\title{
Fatores determinantes na adesão à vacina contra influenza em pessoas idosas de um município do interior de Mato Grosso do Sul
}

\author{
Determining factors in adherence to influenza vaccination in older adults living in a city of the \\ state of Mato Grosso do Sul
}

Humberta Correia Silva Azambuja' $\mathbb{D}$ Mariana Ferreira Carrijoº

Sofia Cristina lost Pavarini ${ }^{3}$ (D) Tatiana Carvalho Reis Martins ${ }^{1,2}$ (D) Bruna Moretti Luchesi, ${ }^{1,2}$

\section{Resumo}

Objetivo: Identificar os fatores sociodemográficos, de saúde e de conhecimento sobre a vacinação relacionados à adesão de pessoas idosas à vacina contra influenza no ano de 2019, em um município do interior de Mato Grosso do Sul, Brasil. Método: Estudo quantitativo e transversal, realizado com 172 indivíduos com 60 anos ou mais, usuários de serviços de saúde e convivência. Aplicou-se um questionário que contemplou dados sociodemográficos, de saúde e de conhecimento sobre vacinação, além da questão sobre a adesão vacinal na campanha de 2019. Utilizou-se regressão log-linear e redes bayesianas de aprendizado para analisar os dados. Resultados: Houve predomínio de pessoas idosas do sexo feminino, com companheiro, entre 60-69 anos de idade e ensino fundamental. A taxa de adesão vacinal contra influenza foi 91,28\% em 2019. Os idosos com doenças neuropsiquiátricas, que costumavam receber a vacina anualmente e que tinham recebido nos anos de 2018, 2016 e 2015 tiveram maior prevalência de adesão vacinal em 2019 no modelo de regressão. As redes bayesianas para adesão vacinal em 2019 evidenciaram que saber que existe o Programa Nacional de Imunização leva a pessoa idosa a confiar no mesmo, o que culmina na adesão anual à vacina. Conclusão: Os profissionais de saúde e meios de comunicação devem fornecer informações válidas para que as pessoas conheçam e confiem no Programa Nacional de Imunização. O trabalho de orientação dos idosos para vacinação contra influenza deve ser anual, já que é importante que o idoso receba a vacina todos os anos para que mantenha a adesão vacinal.

\footnotetext{
Universidade Federal de Mato Grosso do Sul, Programa de Pós-Graduação em Enfermagem, Três Lagoas, MS, Brasil.

2 Universidade Federal de Mato Grosso do Sul, Curso de Graduação em Medicina, Três Lagoas, MS, Brasil.

3 Universidade Federal de São Carlos, Programa de Pós-Graduação em Enfermagem, Programa de PósGraduação em Gerontologia, São Carlos, SP, Brasil.
}

Financiamento da pesquisa: Coordenação de Aperfeiçoamento de Pessoal de Nível Superior Brasil (CAPES) - Código de Financiamento 001; Universidade Federal de Mato Grosso do Sul (UFMS).

Os autores declaram não haver conflito na concepção deste trabalho.

Palavras-chave:

Cobertura Vacinal. Fatores

Epidemiológicos. Idoso.

Vacinas contra Influenza. 


\section{Abstract}

Objective: To identify the sociodemographic, health and knowledge factors related to the adherence to influenza vaccination in older adults in 2019, in a municipality of the state of Mato Grosso do Sul, Brazil. Methods: This is a quantitative and cross-sectional study, carried out with 172 older adults users of health and coexistence services. A questionnaire was applied that covered sociodemographic, health and knowledge about vaccination issues, in addition to vaccination adherence in the 2019 campaign. Log-linear regression and Bayesian networks were used to analyze the data. Results: There was a predominance of women, with a partner, between 60-69 years of age and elementary school. The adherence rate was $91.28 \%$ in 2019 . Older adults with neuropsychiatric diseases, who used to get the vaccine annually and who had gotten it in the years of 2018, 2016 and 2015 had a higher prevalence of adherence to influenza vaccination in 2019. Bayesian networks to the adherence to influenza vaccination in 2019 showed that knowing that the National Immunization Program exists leads to trust in it, which leads the older adults to get the vaccine annually. Conclusion: Health professionals and the media must provide valid information so that people know and trust the National Immunization Program. The work of guiding older people about vaccination against influenza should be annual, as it is important that older adults are vaccinated every year to maintain vaccine adherence.

Keywords: Vaccination Coverage. Epidemiologic Factors. Aged. Influenza Vaccines.

\section{INTRODUÇÃO}

A influenza é uma infecção respiratória aguda causada por subtipos do vírus influenza, que são responsáveis por epidemias sazonais, circulam no mundo todo e atingem pessoas de todas as idades. Alguns grupos apresentam maior risco de serem afetados pelo vírus, e dentre esses encontram-se as pessoas idosas ${ }^{1}$.

Os sintomas da doença são febre de início repentino, tosse (normalmente seca), dores de cabeça e muscular, coriza e mal-estar, os quais podem se resolver espontaneamente em uma semana, mas também podem evoluir para complicações, que são mais frequentes em indivíduos com doenças cardíacas e respiratórias, idosos e imunocomprometidos ${ }^{1}$. As pessoas idosas, portanto, são mais vulneráveis tanto pelas alterações fisiológicas da idade, como pela presença de comorbidades associadas, tornando-as mais frágeis e suscetíveis a morbimortalidade por gripe e infecções secundárias ${ }^{2}$.

A forma mais eficaz de prevenção contra influenza no mundo é a vacinação ${ }^{1}$. No Brasil ela foi introduzida pelo Programa Nacional de Imunização (PNI) em 1999 por meio de campanhas anuais, que objetivavam a vacinação de, no mínimo, 70\% dos idosos a partir de 65 anos de idade. No ano 2000 foram incluídos idosos a partir de 60 anos. Em 2008, a meta de cobertura vacinal passou a ser de $80 \%$, e em 2017 , de $90 \%$, a qual está mantida até os dias atuais ${ }^{3,4}$.

O Brasil tem alcançado a meta de vacinação anual, sendo que em 2018 a cobertura vacinal para influenza em idosos foi de $97,2 \%$, em 2019 de 99,4\% e em 2020 , de $120,7 \%{ }^{5}$. Porém, devido às coberturas vacinais acima de $100 \%$, os dados utilizados para o cálculo podem estar defasados, já que estudos sobre a temática evidenciaram que alguns idosos não aderem às campanhas anuais de vacinação ${ }^{6-12}$. $\mathrm{O}$ fato de um idoso decidir ou não se vacinar pode estar relacionado a diversos aspectos, que podem ser pessoais e até culturais.

A maioria dos estudos desenvolvidos no Brasil sobre o tema é anterior à epidemia de gripe que aconteceu no país no ano de 2016. Características como ter doenças crônicas, ser praticante de atividades físicas, ter companheiro, receber orientações de profissionais de saúde e ter frequentado uma consulta médica nos últimos 12 meses contribuem para a adesão vacinal ${ }^{2,6-12}$. Com relação à idade, resultados contraditórios são encontrados na literatura. A maioria das investigações aponta para maior adesão em pessoas idosas com idade mais elevada ${ }^{2,6,8,9,12,13}$. Um estudo realizado com 286 indivíduos idosos de 
Fortaleza (CE) mostrou maior aceitação entre os mais jovens ${ }^{11}$, e outro conduzido com 1.043 pessoas idosas de São Paulo (SP) não identificou resultados significativos para idade ${ }^{10}$. Uma pesquisa mostrou que não existem iniquidades socioeconômicas com relação à adesão vacinal, e que os principais motivos para a não adesão são falta de confiança na vacina e medo dos efeitos adversos?

$\mathrm{Na}$ China, ser do sexo feminino, ter doença crônica, participar de atividades comunitárias e receber recomendações de provedores de cuidados de saúde foram associados à maior vacinação, evidenciando a importância da disseminação de conhecimentos sobre a necessidade da vacina ${ }^{14}$. $\mathrm{Na}$ Arábia Saudita, as pessoas idosas com maior nível de escolaridade apresentaram maior adesão vacinal $^{15}$. Em Israel, a equipe de saúde influenciou no aumento das taxas de vacinação por fornecer informações seguras ${ }^{16}$.

Uma revisão sistemática concluiu que a vacinação contra influenza sazonal é influenciada por determinantes sociais estruturais, como sexo, idade, estado civil, escolaridade, entre outros; intermediários, como local de residência, crenças comportamentais, influências sociais, fontes de informação; e relacionados à saúde, como acessibilidade, conhecimento sobre vacinação e conselho de profissionais de saúde ${ }^{17}$. Em outra revisão, evidenciou-se que barreiras psicológicas (utilidade, percepção de risco, benefício social, atitude, experiência, comportamentos passados e conhecimento), físicas (consumo de álcool e cigarro, inatividade física e condições médicas), contextuais (falta de acesso e de interação com serviços de saúde, receber recomendações de profissionais) e sociodemográficas (idade, sexo, raça, estado civil) podem levar à recusa da vacina ${ }^{18}$.

Portanto, a literatura mostra que os fatores relacionados à adesão vacinal contra influenza em pessoas idosas variam. No Brasil, a cobertura vacinal para influenza foi alta após a epidemia de $2016^{5}$, e entender os fatores relacionados à adesão dos idosos após essa epidemia pode contribuir para aumentar ainda mais essas taxas. O presente estudo teve como objetivo identificar as variáveis sociodemográficas, de saúde e de conhecimento sobre a vacinação relacionadas à adesão das pessoas idosas à vacina contra influenza no ano de 2019, em um município do interior de Mato Grosso do Sul, Brasil. Como hipóteses, espera-se que a adesão vacinal contra influenza em pessoas idosas esteja relacionada às variáveis sociodemográficas, de saúde e de conhecimento sobre vacinação.

\section{MÉTODO}

Trata-se de um estudo descritivo, transversal, de abordagem quantitativa, realizado mediante aplicação de um questionário na população idosa atendida em serviços de saúde ou de convivência na cidade de Três Lagoas (MS), Brasil. Em 2019, o município possuía população estimada de 121.388 habitantes e havia 16 Unidades Básicas de Saúde (UBS) na cidade. $\mathrm{O}$ atendimento de média complexidade é realizado mediante encaminhamento dessas UBS para o Centro de Especialidades Médicas (CEM) e para as Clínicas do Idoso, da Criança, da Mulher e de Ortopedia. $\mathrm{Na}$ cidade existem duas associações de aposentados, uma de trabalhadores da educação e uma de trabalhadores de companhias energéticas, as quais oferecem atividades de convivência para os afiliados.

Os critérios de inclusão foram ter idade igual ou superior a 60 anos, ser atendido em um serviço de saúde ou de convivência do município e ser capaz de compreender as questões da entrevista (avaliada pela percepção do entrevistador). O critério de exclusão foi não residir no município.

O tamanho da amostra foi determinado de acordo com a fórmula de estimativa de proporção em estudo de população finita. Foi utilizado o nível de significância de 10\% (alfa=0,10), um erro amostral de $5 \%(e=0,05)$, uma estimativa de proporção de $80 \%(p=0,80)$, considerando que a cobertura vacinal em pessoas idosas estava acima desse valor, e um tamanho de população finita $\mathrm{N}=10.067$, que corresponde ao total de idosos do município de acordo com o censo de 2010. A amostra mínima era de 171 participantes. Após a coleta houve o cálculo da função poder para investigar a plausibilidade do tamanho amostral e não houve descaracterização e inconsistência. 
Os idosos foram selecionados em diferentes serviços, escolhidos por serem locais onde há alto fluxo de atendimento de pessoas idosas, sendo uma UBS na região central da cidade, a Clínica do Idoso e o CEM. Também foram incluídas as duas associações de aposentados, nas quais o público é predominantemente idoso.

Nesses locais, as pessoas idosas eram abordadas de forma aleatória pelas pesquisadoras, enquanto aguardavam atendimento ou participavam das atividades oferecidas nos locais, e eram convidadas a participar da pesquisa. As entrevistas aconteceram em diferentes períodos, e foi estimada a coleta de aproximadamente 50 participantes em cada local. Foram abordados 183 idosos, sendo que 11 recusaram a participação, resultando em uma amostra de 172 participantes (taxa de resposta 93,9\%). Os locais foram uma UBS ( $n=53)$; o CEM $(n=40)$, a Clínica do Idoso ( $n=50)$, e as duas associações de aposentados $(n=29)$. As associações foram os últimos locais visitados e a coleta cessou quando a amostra mínima de participantes foi atingida.

Foi aplicado um questionário por dois entrevistadores treinados, em local reservado, entre janeiro e março de 2020. O questionário foi desenvolvido pelas pesquisadoras, de acordo com manuais de vacinação do Ministério da Saúde, da Sociedade Brasileira de Imunização e com dados de adesão e não adesão à vacina contra influenza da literatura ${ }^{2-4,6-13,19-23}$. O instrumento passou por uma validação de face, um subtipo da validação de conteúdo, em que outras pessoas analisam o conteúdo para verificar se ele realmente proporciona o que o pesquisador deseja avaliar ${ }^{24}$. Três profissionais fizeram a validação, duas da área de gerontologia e uma da área de vacinação. O instrumento foi adaptado de acordo com as sugestões e foi aplicado em cinco idosos para verificar a compreensão e facilidade de aplicação. Todos os idosos compreenderam as questões, e a versão final do instrumento continha os seguintes dados:

- Caracterização sociodemográfica: sexo (masculino, feminino), idade (60-69, 70-79, 80 anos ou mais), escolaridade (não alfabetizado, fundamental/ primário, médio/ secundário, superior/ pós- graduação, não sabe/ não respondeu), situação conjugal (com companheiro, sem companheiro) e religião (católico, evangélico, espírita, outra, nenhuma, não sabe/ não respondeu).

- Caracterização de saúde e de atividades sociais: uso diário de medicamentos (sim, não); condições autorreferidas (sim, não), sendo elas doenças cardiovasculares (como hipertensão arterial sistêmica e insuficiência cardíaca), doenças respiratórias (como insuficiência respiratória, doença pulmonar obstrutiva crônica e bronquite), doenças endócrinas (como distúrbios da tireoide e diabetes mellitus), doenças neurológicas (como doença de Alzheimer e doença de Parkinson), doenças osteoarticulares (como osteoartrose e osteoartrite), doenças neuropsiquiátricas (como depressão, ansiedade, síndrome do pânico e transtorno bipolar), doenças gastrointestinais (como gastrite e úlcera gástrica), doenças urinárias (como insuficiência renal e nefrite) e outras doenças; prática de atividades físicas (sim, não); e participação em atividades sociais ("Participa de atividades sociais (centro comunitário, igreja ou grupos)?" - sim, não). Caso o participante mencionasse ter outra doença que não constava na lista de exemplos, mas que se enquadrava nas categorias acima, o pesquisador marcava como "sim". Caso a doença não se encaixasse nas categorias, era marcada como "outra".

- Questões relacionadas à vacinação contra influenza: "Você sabia que o Brasil possui um Programa Nacional de Imunização?" (sim, não, não respondeu), "Se sim, você confia no PNI do Brasil?" (sim, não); "Segundo o seu conhecimento, a vacinação contra gripe para pessoas $\geq 60$ anos é indicada no Brasil?" (sim, não); “Já apresentou alguma reação adversa à vacina contra gripe?" (sim, não, não sabe ou não respondeu); "Costuma receber a vacina anualmente?” (sim, não); "Recebeu em 2019? Em 2018? Em 2017? Em 2016? E em 2015?” (sim, não). Essas questões foram feitas de acordo com a idade do idoso no referido ano. Por exemplo, caso ele não tivesse 60 anos completos em 2015, a pergunta para este ano não era feita. 
Os dados foram digitados, validados por dupla digitação e armazenados em planilhas eletrônicas. Foram construídas tabelas com medidas descritivas, organizadas e descritas em termos de frequência e percentual. Foi feito o cálculo da razão de prevalência, utilizando modelo de regressão log-linear ${ }^{25}$.

Utilizou-se redes bayesianas de aprendizado, especificamente algoritmo IAMB-Incremental Association Markov Blanket ${ }^{26}$ para estudar caminhos e inter-relações entre a adesão vacinal em 2019 e: saber que existe o PNI, confiar no PNI, saber que a vacina é indicada para idosos, ter o costume de receber a vacina anualmente, ter tido algum evento adverso anterior e ter tomado a vacina nos anos de 2015 a 2018. Elas se baseiam em técnicas de aprendizado e detecção da estrutura de dados, gerando e analisando a relação probabilística implicada pela propriedade de probabilidade e independência condicional. Depois de estruturadas as relações, é possível construir um gráfico acíclico direcionado (Directed Acyclic Graph - DAG) que mostre os caminhos possíveis e associações entre as variáveis estudadas ${ }^{27}$.

Em todas as análises considerou-se um nível de significância de 5\%. Após a coleta de dados, foi realizada uma análise de poder de explicação da amostra coletada a posteriori, considerando o alfa utilizado nas análises principais $(0,05)$, e o mesmo foi de $83 \%$.

A pesquisa está de acordo com a Resolução $n^{\circ}$ 466/2012 e a Resolução no 510/2016, e foi aprovada pelo Comitê de Ética e Pesquisa com Seres Humanos da Universidade Federal de Mato Grosso do Sul (parecer $\mathrm{n}^{\circ}$ 4.216.102). Os dados foram coletados após a leitura e assinatura do Termo de Consentimento Livre e Esclarecido pelos participantes, em duas vias.

\section{RESULTADOS}

A Tabela 1 evidencia os dados de caracterização sociodemográfica, de saúde e social dos participantes da amostra total e de acordo com a adesão vacinal contra influenza em 2019. Também é apresentada a razão de prevalência para as variáveis estudadas. A maior proporção da amostra era de pessoas idosas do sexo feminino, entre 60-69 anos de idade, com ensino fundamental, com companheiro e católica. A maioria fazia uso diário de medicamentos, não praticava atividades físicas, não participava de atividades sociais; e as doenças mais prevalentes foram as cardiovasculares, endócrinas e osteomusculares.

$\mathrm{Na}$ Tabela 2, são apresentados os dados das variáveis relacionadas à vacinação de acordo com a adesão vacinal para influenza e a razão de prevalência. A taxa de adesão à vacina na campanha de 2019 foi de 91,28\%. A maioria conhece o PNI e confia no mesmo, sabe que a vacina é indicada para idosos, e costuma receber a vacina anualmente.

O modelo de regressão mostrou que os grupos adesão e não adesão vacinal diferem quanto às doenças neuropsiquiátricas. Os indivíduos que relataram ter alguma doença neuropsiquiátrica tiveram maior prevalência de adesão em relação aos que não tinham essas doenças. Além disso, as análises mostraram que as pessoas idosas que costumam receber a vacina anualmente e que tinham tomado em 2018, 2016 e 2015 tinham maior prevalência de adesão à vacina no ano de 2019. 
Tabela 1. Caracterização sociodemográfica, de saúde e social das pessoas idosas participantes $(\mathrm{N}=172)$ de acordo com a adesão vacinal contra influenza em 2019 e razão de prevalência para as variáveis estudadas. Três Lagoas, MS, 2020.

\begin{tabular}{|c|c|c|c|c|c|}
\hline \multirow[t]{2}{*}{ Variáveis } & Total $(\mathrm{N}=172)$ & $\begin{array}{l}\text { Adesão vacinal } \\
(\mathrm{n}=157)\end{array}$ & $\begin{array}{l}\text { Não adesão } \\
\text { vacinal }(n=15)\end{array}$ & $\begin{array}{l}\mathrm{RP} \\
(\mathrm{IC} 95 \%)\end{array}$ & $p$ \\
\hline & $\mathrm{n}(\%)$ & $\mathrm{n}(\%)$ & $\mathrm{n}(\%)$ & & \\
\hline \multicolumn{6}{|l|}{ Sociodemográficas } \\
\hline Masculino & $61(35,47)$ & $56(35,67)$ & $5(33,33)$ & 1,0 & \\
\hline Feminino & $111(64,53)$ & $101(64,33)$ & $10(66,67)$ & $1,01(0,92-1,11)$ & 0,855 \\
\hline \multicolumn{6}{|l|}{ Idade (anos) } \\
\hline $60-69$ & $95(55,23)$ & $87(55,41)$ & $8(53,33)$ & 1,0 & \\
\hline $70-79$ & $65(37,79)$ & $60(38,22)$ & $5(33,33)$ & $1,01(0,92-1,11)$ & 0,867 \\
\hline 80 ou mais & $12(6,98)$ & $10(6,37)$ & $2(13,44)$ & $0,91(0,70-1,18)$ & 0,477 \\
\hline \multicolumn{6}{|l|}{ Escolaridade } \\
\hline Não alfabetizado & $45(26,16)$ & $40(25,48)$ & $5(33,33)$ & 1,0 & \\
\hline Fundamental/Primário & $75(43,61)$ & $69(43,95)$ & $6(40,00)$ & $1,03(0,91-1,17)$ & 0,598 \\
\hline Médio/Secundário & $25(14,53)$ & $22(14,01)$ & $3(20,00)$ & $0,99(0,84-1,18)$ & 0,956 \\
\hline Superior/Pós-graduação & $26(15,12)$ & $25(15,92)$ & $1(6,67)$ & $1,08(0,95-1,23)$ & 0,232 \\
\hline Não sabe/ Não respondeu & $1(0,58)$ & $1(0,64)$ & $0(0,00)$ & - & - \\
\hline \multicolumn{6}{|l|}{ Estado civil } \\
\hline Com companheiro & $91(52,91)$ & $72(45,86)$ & $9(60,00)$ & 1,0 & \\
\hline Sem companheiro & $81(47,09)$ & $85(54,14)$ & $6(40,00)$ & $0,95(0,87-1,05)$ & 0,303 \\
\hline \multicolumn{6}{|l|}{ Religião } \\
\hline Católico & $97(56,40)$ & $89(56,67)$ & $8(53,33)$ & Não estimado & \\
\hline Evangélico & $59(34,30)$ & $52(33,12)$ & $7(46,67)$ & & \\
\hline Espírita & $10(5,82)$ & $10(6,37)$ & $0(0,00)$ & & \\
\hline Outra & $3(1,74)$ & $3(1,92)$ & $0(0,00)$ & & \\
\hline Nenhuma/ Não respondeu & $3(1,74)$ & $3(1,92)$ & $0(0,00)$ & & \\
\hline \multicolumn{6}{|l|}{ Saúde e sociais } \\
\hline Uso de medicamentos & $158(91,86)$ & $144(91,72)$ & $14(93,33)$ & $0,98(0,84-1,14)$ & 0,811 \\
\hline Doenças cardiovasculares & $121(70,35)$ & $108(68,79)$ & $13(86,67)$ & $1,07(0,99-1,17)$ & 0,082 \\
\hline Doenças respiratórias & $14(8,14)$ & $13(8,28)$ & $1(6,67)$ & $0,98(0,84-1,14)$ & 0,811 \\
\hline Doenças endócrinas & $74(43,02)$ & $69(43,95)$ & $5(33,33)$ & $0,96(0,88-1,05)$ & 0,415 \\
\hline Doenças neurológicas & $8(4,65)$ & $8(5,10)$ & $0(0,00)$ & Não estimado & \\
\hline Doenças osteoarticulares & $50(29,07)$ & $45(28,66)$ & $5(33,3)$ & $1,02(0,91-1,13)$ & 0,710 \\
\hline Doenças neuropsiquiátricas & $39(22,67)$ & $38(24,20)$ & $1(6,67)$ & $0,92(0,85-0,99)$ & 0,030 \\
\hline Doenças gastrointestinais & $21(12,21)$ & $19(12,10)$ & $2(13,33)$ & $1,01(0,87-1,17)$ & 0,893 \\
\hline Doenças urinárias & $7(4,07)$ & $7(4,46)$ & $0(0,00)$ & Não estimado & \\
\hline Outras doenças & $31(18,02)$ & $31(19,75)$ & $0(0,00)$ & Não estimado & \\
\hline Prática de atividades físicas & $77(44,77)$ & $69(43,95)$ & $8(53,33)$ & $1,03(0,94-1,14)$ & 0,493 \\
\hline Participação em atividades sociais & $70(40,70)$ & $65(41,40)$ & $5(33,33)$ & $1,03(0,94-1,13)$ & 0,532 \\
\hline
\end{tabular}

Fonte: tabela elaborada pelos próprios autores. RP: razão de prevalência; categoria de referência - não adesão; IC95\%: intervalo de confiança de $95 \%$. 
Tabela 2. Caracterização da vacinação das pessoas idosas participantes $(\mathrm{N}=172)$ de acordo com a adesão vacinal contra influenza em 2019 e razão de prevalência para as variáveis estudadas. Três Lagoas, MS, 2020.

\begin{tabular}{|c|c|c|c|c|c|}
\hline Variáveis & Total $(\mathrm{N}=172)$ & $\begin{array}{l}\text { Adesão vacinal } \\
(\mathrm{n}=157)\end{array}$ & $\begin{array}{l}\text { Não adesão } \\
\text { vacinal }(n=15)\end{array}$ & $\begin{array}{l}\mathrm{RP} \\
(\mathrm{IC} 95 \%)\end{array}$ & $p$ \\
\hline Vacinação & $\mathrm{n}(\%)$ & $\mathrm{n}(\%)$ & $\mathrm{n}(\%)$ & & \\
\hline \multicolumn{6}{|l|}{ Conhece PNI } \\
\hline $\operatorname{Sim}$ & $121(70,35)$ & $110(70,06)$ & $11(73,34)$ & 1,00 & \\
\hline Não & $27(15,70)$ & $25(15,92)$ & $2(13,33)$ & $1,02(0,90-1,15)$ & 0,765 \\
\hline Não respondeu & $24(13,95)$ & $22(14,02)$ & $2(13,33)$ & $0,99(0,84-1,16)$ & 0,903 \\
\hline Confia no PNI ( $n=121)$ & $108(86,26)$ & $100(90,91)$ & $8(72,73)$ & $0,83(0,61-1,12)$ & 0,229 \\
\hline \multicolumn{6}{|l|}{ Sabe que a vacina é indicada para idosos } \\
\hline Sim & $165(95,93)$ & $153(97,45)$ & $12(80,00)$ & Não estimado & \\
\hline Não & $1(0,58)$ & $1(0,64)$ & $0(0,00)$ & & \\
\hline Não respondeu & $6(3,49)$ & $3(1,91)$ & $3(20,00)$ & & \\
\hline \multicolumn{6}{|l|}{ Reação adversa anterior } \\
\hline $\operatorname{Sim}$ & $25(14,53)$ & $22(14,01)$ & $3(20,00)$ & 1,0 & \\
\hline Não & $142(82,56)$ & $131(83,44)$ & $11(73,33)$ & $1,05(0,90-1,22)$ & 0,543 \\
\hline Não respondeu/ Não sabe & $5(2,91)$ & $4(2,55)$ & $1(6,67)$ & - & - \\
\hline Costuma receber a vacina anualmente & $157(91,28)$ & $154(98,09)$ & $3(20,00)$ & $0,20(0,07-0,56)$ & 0,002 \\
\hline Recebeu em $2018(n=169)$ & $141(83,43)$ & $138(89,61)$ & $3(20,00)$ & $0,58(0,42-0,80)$ & 0,001 \\
\hline Recebeu em 2017 ( $n=161)$ & $129(80,12)$ & $129(87,76)$ & $0(0,00)$ & Não estimado & \\
\hline Recebeu em $2016(n=157)$ & $120(76,43)$ & $119(83,22)$ & $1(7,14)$ & $0,65(0,51-0,83)$ & $<0,001$ \\
\hline Recebeu em 2015 (n=154) & $114(74,03)$ & $113(80,71)$ & $1(7,14)$ & $0,68(0,55-0,84)$ & $<0,001$ \\
\hline
\end{tabular}

Fonte: tabela elaborada pelos próprios autores; RP: razão de prevalência; categoria de referência - não adesão; IC95\%: intervalo de confiança de 95\%; PNI: Programa Nacional de Imunização.

Adicionalmente, as Redes Bayesianas de aprendizado (Figura 1), mostraram os caminhos para adesão à vacina em 2019, evidenciando que saber que existe o PNI leva a confiar no mesmo, o que leva os idosos a receber a vacina anualmente, inclusive em 2019. Saber que a vacina é indicada para idosos e ter tido algum evento adverso anterior não foram inter-relacionados à vacinação. 


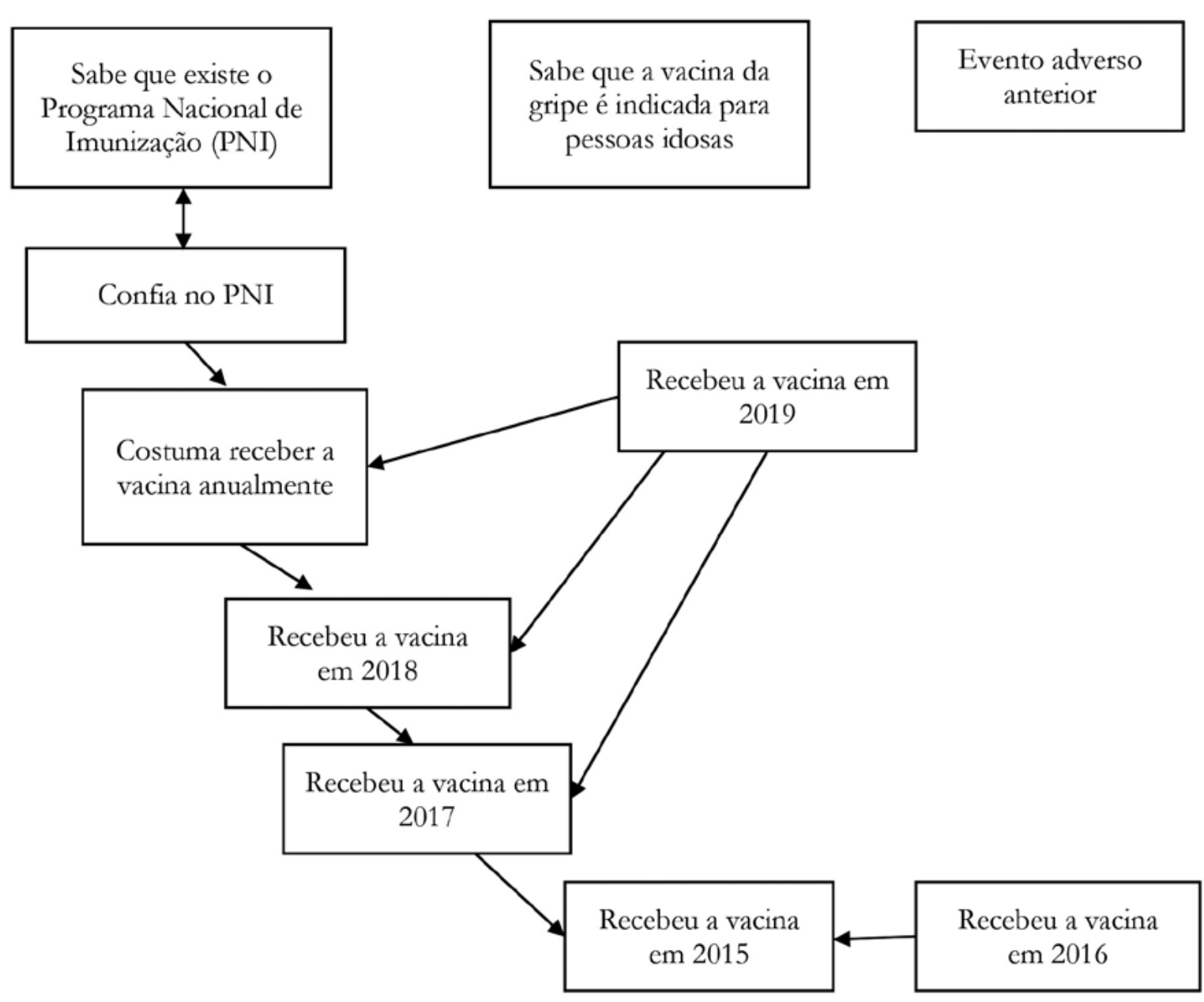

Figura 1. Gráficos acíclicos direcionais, resultado da rede bayesiana de aprendizado, para as variáveis de adesão vacinal em pessoas idosas no ano de 2019. Três Lagoas, MS, 2020.

\section{DISCUSSÃO}

A cobertura vacinal contra a gripe na amostra estudada foi de $91,28 \%$ no ano de 2019 , sendo que aqueles com doenças neuropsiquiátricas, que costumavam receber a vacina anualmente e que tinham recebido nos anos de 2018, 2016 e 2015 tiveram maior prevalência de adesão vacinal. Saber que existe o PNI leva a pessoa idosa a confiar no mesmo, o que resulta na vacinação anual, inclusive em 2019.

Dados do Sistema de Informações do PNI (SIPNI) mostram que a cobertura em 2019 no Brasil foi 99,4\%, e no município de Três Lagoas (MS), $118,4 \%$, acima da meta de $90 \%$ estabelecida pelo Ministério da Saúde 5 .
Estudos sobre a adesão vacinal em pessoas idosas no Brasil encontraram as prevalências de $58,7 \%$ em Timon (MA) ${ }^{19}, 62,6 \%$ em Campinas (SP) 7 , 62,9\% em Fortaleza (CE) ${ }^{11}, 66,9 \%$ em cidades do estado de São Paulo ${ }^{6}, 71,0 \%$ em Pelotas (RS) ${ }^{20}, 71,9 \%$ em Jundiaí (SP) ${ }^{21}, 72,6 \%$ em uma amostra representativa de idosos brasileiros ${ }^{12}, 73,8 \%$ em São Paulo capital ${ }^{8}$, $74,4 \%$ em Campinas (SP) ${ }^{22}, 74,6 \%$ em Cambé (PR) ${ }^{2}$, 79,7\% em 1043 idosos do Estudo Saúde, Bem-Estar e Envelhecimento ${ }^{10}$ e $82,4 \%$ em Teresina (PI) ${ }^{23}$. Por fim, em uma investigação com 5221 idosos de 70 municípios do Brasil, utilizando dados do Estudo Longitudinal da Saúde dos Idosos (ELSI-Brasil), identificou-se cobertura de 73,0\% ${ }^{9}$. Ressalta-se que todos os estudos citados foram desenvolvidos antes da epidemia de gripe de 2016. A adesão encontrada 
na atual investigação foi a maior em comparação às outras investigações, o que pode estar relacionado a um viés de seleção da amostra, mas também pode ser um reflexo do aumento das coberturas vacinais a nível nacional, que passaram de 89,1\% em 2015, para 99,4\% em $2019^{5}$. Acredita-se que o investimento em campanhas nacionais de imunização, bem como o fato de a população estar mais acostumada com as campanhas anuais em idosos podem contribuir com o aumento das coberturas vacinais.

Ressalta-se que os dados disponíveis no SI-PNI são calculados pela fórmula "(número de doses aplicadas em um determinado ano/ população de pessoas idosas no local no mesmo ano) x 100". Para cálculo das coberturas para pessoas idosas, o denominador utilizado no ano de 2019 correspondia à uma estimativa da população publicada pelo Instituto Brasileiro de Geografia e Estatística em 2012, ou seja, as informações sobre a cobertura vacinal podem estar superestimadas. Para Três Lagoas (MS), o número de pessoas idosas utilizado no cálculo foi 10.406 , e o número de pessoas idosas vacinadas em 2019 foi $12.319^{5}$. Com o envelhecimento populacional, o número de idosos deve estar acima do que foi utilizado para cálculo, indicando que os dados oficiais não condizem com a realidade. Além disso, tanto os dados autorreferidos do presente estudo, como os das investigações anteriores citadas, podem estar sujeitos ao viés de memória dos indivíduos idosos. Porém, para a vacina contra influenza que é anual e de ampla escala, os dados de vacinação não são inseridos nos sistemas de informação de forma individualizada, ou seja, não é possível confirmar se uma pessoa idosa que relatou ter tomado a vacina, realmente tomou.

Ao contrário de estudos anteriores que mostraram relação entre adesão vacinal e sexo, idade, escolaridade, estado civil e religião $\mathrm{O}^{2,6-9,11-13,20,22}$, a presente pesquisa não identificou essa relação. Também não foi identificada relação entre adesão e uso de medicamentos, prática de atividades físicas e participação em atividades sociais, como relatado anteriormente ${ }^{6,11,22}$.

A Política Nacional de Atenção Básica de 2017 incentiva o aumento da cobertura da APS em todo o país $^{28}$, o que pode culminar no maior envolvimento das equipes multiprofissionais, aproximação com a população no território, e na expansão da oferta da vacina. Além disso, propostas como o Programa Saúde na Hora que amplia o funcionamento da UBS para um horário estendido ${ }^{29}$ e postos de vacinação extramuros, como shoppings, metrôs, rodoviárias, aumentam a possibilidade de os usuários terem acesso ao imunobiológico, independente de características sociodemográficas e de saúde; o que pode ter contribuído para a cobertura vacinal encontrada no presente estudo. Porém, os dados devem ser vistos com cautela, por serem referentes a uma amostra de pessoas idosas de um único município brasileiro.

A relação entre doenças crônicas e cobertura vacinal para influenza já foi descrita anteriormente $e^{7-9,11-14,17}$. $\mathrm{Na}$ presente investigação, as doenças neuropsiquiátricas foram as únicas que estiveram relacionadas à adesão vacinal. Quem relatou ter alguma doença desse grupo, tinha maior prevalência de adesão. $\mathrm{Na}$ Coreia do Sul, não foi encontrada relação entre humor deprimido e estresse, e a cobertura vacinal para influenza em idosos ${ }^{30}$. Em contrapartida, outra investigação evidenciou que pessoas idosas identificadas com solidão crônica visitavam mais o médico do que indivíduos idosos não solitários ${ }^{31}$, o que pode colaborar para a adesão vacinal. Porém, a relação entre transtornos psiquiátricos e vacinação precisa ser melhor investigada em estudos posteriores, incluindo qual transtorno o indivíduo possui.

As análises evidenciaram que pessoas idosas que costumam receber a vacina anualmente, e que tinham recebido em 2018, 2016 e 2015 tinham maior prevalência de adesão à vacina no ano de 2019. As Redes Bayesianas de aprendizado também suportam esses dados, mostrando que saber que existe o PNI leva o idoso a confiar no mesmo, que leva os idosos a receber a vacina anualmente. Portanto, o conhecimento e a confiança sobre o PNI foram fatores determinantes para a adesão vacinal. Uma revisão sistemática descreveu variáveis sociodemográficas, físicas, comportamentos passados (não ter tomado a vacina anteriormente) e falta de confiança na vacina como fatores que levam à recusa da vacina ${ }^{18}$. O presente estudo está em consonância com os resultados da revisão quando evidencia relação entre ter tomado a vacina anteriormente, 
confiança no programa de imunização e adesão vacinal. Barreiras potenciais da vacinação, como a falta de confiança, precisam ser transpostas para aumentar a conscientização e a aceitação à vacina.

Um estudo grego evidenciou que crenças relacionadas à influência de outras pessoas no comportamento e atos como recomendações dos médicos, farmacêuticos, familiares e amigos; estiveram relacionadas à adesão vacinal ${ }^{32}$. Os autores sugerem que o suporte informacional e a educação em saúde são essenciais para o aumento da cobertura vacinal em pessoas idosas, e os profissionais de saúde possuem um papel central, pois podem oferecer informações claras, abrangentes e verdadeiras sobre a vacina $^{32}$. Em Portugal, o maior uso de serviços de saúde, ter aferido a pressão arterial nos últimos 12 meses no serviço e ter visitado o médico de família nas últimas quatro semanas foram associados à adesão vacinal ${ }^{33}$, reforçando a importância de se aproveitar o contato do paciente com o serviço falar da importância da vacinação.

As propagandas e orientações referentes a divulgação de datas, locais e horários das campanhas nacionais de imunização pela mídia podem incentivar a vacinação da população. A Pesquisa Nacional por Amostra de Domicílios Contínua pesquisou o acesso dos brasileiros à Tecnologia da Informação e Comunicação (TIC) e identificou que a proporção de idosos que acessaram a internet subiu de 24,7\% em; 2016, para 31,1\% em 2017, evidenciando o maior aumento proporcional entre os grupos etários ${ }^{34}$.

Ter apresentado algum evento adverso anterior não foi inter-relacionado à vacinação em 2019, diferentemente de uma revisão que evidenciou que a decisão de se vacinar ou não contempla a gravidade percebida da doença, a probabilidade de adquiri-la, e o risco de eventos adversos pós-vacinação ${ }^{18}$. Outras investigações relatam que o medo dos efeitos adversos pós-vacinais pode contribuir para a não adesão ${ }^{2,9,21,35}$, porém, esses estudos foram desenvolvidos antes da epidemia de 2016. Uma hipótese para esse dado não ter sido evidenciado no presente estudo é que, com a epidemia, mais informação sobre os eventos adversos vacinais foram disseminadas, reduzindo o medo. Porém, o tema carece de novas pesquisas para ser melhor compreendido.
O presente estudo possui algumas limitações: por se tratar de um delineamento transversal, não é possível inferir a causalidade; apesar do esforço para incluir participantes idosos de diferentes pontos de atendimento do município, os dados não podem ser generalizados, por se tratar de uma amostra de conveniência composta por indivíduos que frequentavam serviços de saúde e de convivência de um único município, os quais são mais propensos ao autocuidado; algumas informações autorreferidas como a presença de doenças, o uso de medicamentos e a adesão vacinal estão sujeitas ao viés de memória dos participantes. Não podemos deixar de citar a escassez de investigações com dados recentes relacionados à vacinação contra influenza em pessoas idosas na literatura nacional, o que restringe a discussão sobre a realidade brasileira. Apesar disso, entende-se que o estudo contribui para o conhecimento atual acerca dos fatores relacionados à adesão vacinal.

\section{CONCLUSÃO}

Houve maior prevalência de adesão vacinal contra influenza nas pessoas idosas com doenças neuropsiquiátricas. A maioria dos participantes conhecia o PNI e confiava no mesmo, sabia que a vacina é indicada para idosos, e costumava receber a vacina anualmente, variáveis que estiveram relacionadas à maior prevalência na adesão vacinal. Não foi identificada relação entre a adesão vacinal e variáveis sociodemográficas.

A educação em saúde na comunidade deve ser conduzida para que as pessoas idosas conheçam o PNI e confiem no mesmo, visando evitar possíveis distorções que diminuam a cobertura vacinal. Destaca-se a importância dos profissionais de saúde como formadores de opinião sobre a vacinação. Além disso, é importante que o idoso receba a vacina todos os anos para que mantenha a adesão vacinal, portanto, o trabalho de orientação e captação do público-alvo deve ser anual. Também reforçamos que aproveitar os meios de comunicação para divulgar informações confiáveis sobre as campanhas nacionais de vacinação pode ser uma ferramenta eficaz de informação e conhecimento.

Editor: Maria Luiza Diniz de Sousa Lopes 


\section{REFERÊNCIAS}

1. World Health Organization. Influenza (Seasonal) [Internet]. Genebra: OMS; 2018 [acesso em 16 jan. 2021];[8 telas]. Disponível em: https://www.who.int/ news-room/fact-sheets/detail/influenza-(seasonal).

2. Campos EC, Sudan LCP, Mattos ED, Fidelis R. Fatores relacionados à vacinação contra a gripe em idosos: estudo transversal, Cambé, Paraná, Brasil. Cad Saúde Pública. 2012;28(5):878-88. Disponível em: https://doi.org/10.1590/S0102-311X2012000500007.

3. Brasil. Ministério da Saúde, Fundação Nacional de Saúde. Informe Técnico: $21^{\text {a }}$ Campanha Nacional de Vacinação do Idoso, 2019 [Internet]. Brasília, DF: MS; 2019 [acesso em 19 jan. 2021]. Disponível em: http:// www.cosemssp.org.br/wp-content/uploads/2019/04/ Informe-21\%C2\%AA-Campanha-Nacional-deVacina \%C3\%A7\%C3\%A3o-contra-a-Influenza-1.pdf.

4. Brasil. Ministério da Saúde, Fundação Nacional de Saúde. Informe Técnico: $22^{a}$ Campanha Nacional de Vacinação do Idoso, 2020 [Internet]. Brasília, DF: MS; 2020 [acesso em 19 jan. 2021]. Disponível em: https:// sbim.org.br/images/files/notas-tecnicas/informetecnico-ms-campanha-influenza-2020-final.pdf.

5. Brasil. Ministério da Saúde. Sistema de Informações do Programa Nacional de Imunizações - Datasus [Internet]. Brasília, DF: MS; 2020 [acesso em 19 jan. 2021]. Disponível em: http://sipni.datasus.gov.br.

6. Francisco PMSB, Donalisio MR, Barros MBA, Cesar CLG, Carandina L, Goldbaum M. Fatores associados à vacinação contra a influenza em idosos. Rev Panam Salud Publica [Internet]. 2006;19(4):25964. Disponível em: https://www.scielosp.org/pdf/ rpsp/2006.v19n4/259-264/pt

7. Francisco PMSB, Barros MBA, Cordeiro MRD. Vacinação contra influenza em idosos: prevalência, fatores associados e motivos da não-adesão em Campinas, São Paulo, Brasil. Cad Saúde Pública [Internet]. 2011;27(3):417-26. Disponível em: https:// doi.org/10.1590/S0102-311X2011000300003

8. Moura RF, Andrade FB, Duarte YAO, Lebrão ML, Antunes JLF. Fatores associados à adesão à vacinação anti-influenza em idosos não institucionalizados, São Paulo, Brasil. Cad Saúde Pública [Internet]. 2015;31(10):2157-68. Disponível em: https://doi. org/10.1590/0102-311X00065414.

9. Sato APS, Antunes JLF, Lima-Costa MFF, de Andrade FB. Influenza vaccine uptake among older adults in Brazil: Socioeconomic equality and the role of preventive policies and public services. J Infect Public Health [Internet]. 2019;13(2):211-5. Disponível em: https://doi.org/10.1016/j.jiph.2019.07.022.
10. Sato APS, Andrade FB, Duarte YAO, Antunes JLF. Vaccine coverage and factors associated with influenza vaccination in the elderly in the city of São Paulo, Brazil: SABE Study 2015. Cad Saúde Pública [Internet]. 2020;36(Suppl 2):e00237419. Disponível em: https://doi.org/10.1590/0102-311x00237419.

11. Victor JF, Gomes GD, Sarmento LR, Soares AMG, Mota FRN, Leite BMB, et al. Factors associated with vaccination against Influenza A (H1N1) in the elderly. Rev Esc Enferm USP [Internet]. 2014;48(1):58-65. Disponível em: https://doi.org/10.1590/S0080623420140000100007.

12. Andrade FB, Sato APS, Moura RF, Ferreira Antunes JL. Correlates of influenza vaccine uptake among community-dwelling older adults in Brazil. Hum Vaccin Immunother [Internet]. 2017;13(1):103-10. Disponível em: https://doi.org/10.1080/21645515.20 16.1228501.

13. Okoli GN, Lam OLT, Racovitan F, Reddy VK, Righolt CH, Neilson C, et al. Seasonal influenza vaccination in older people: a systematic review and meta-analysis of the determining factors. PLoS ONE [Internet]. 2020;15(6):e0234702. Disponível em: https://doi.org/10.1371/journal.pone.0234702.

14. Mo PKH, Lau JTF. Influenza vaccination uptake and associated factors among elderly population in Hong Kong: the application of the Health Belief Model. Health Educ Res [Internet]. 2015;30(5):706-18. Disponível em: https://doi.org/10.1093/her/cyv038.

15. Aloitabi FY, Alhetheel AF, Alluhaymid YM, Alshibani MG, Almuhaydili AO, Alhuqayl TA, et al. Influenza vaccine coverage, awareness, and beliefs regarding seasonal influenza vaccination among people aged 65 years and older in Central Saudi Arabia. Saudi Med J [Internet]. 2019;40(10):10138. Disponível em: https://doi.org/10.15537/ smj.2019.11.24587.

16. Ellen M. Factors that influence influenza vaccination rates among the elderly: nurses' perspectives. J Nurs Manag [Internet]. 2017;26(2):158-66. Disponível em: http://dx.doi.org/10.1111/jonm.12528

17. Nagata JM, Hernández-Ramos I, Kurup AS, Albrecht D, Vivas-Torrealba C, Franco-Paredes C. Social determinants of health and seasonal influenza vaccination in adults $\geq 65$ years: a systematic review of qualitative and quantitative data. BMC Public Health [Internet]. 2013;13(388):2-25. Disponível em: http:// www.biomedcentral.com/1471-2458/13/388. 
18. Schmid P, Rauber D, Betsch C, Lidolt G, Denker, ML. Barriers of Influenza Vaccination Intention and Behavior: a systematic review of Influenza Vaccine Hesitancy, 2005-2016. PLoS ONE [Internet]. 2017;12(1):e0170550. Disponível em: https://doi. org/10.1371/journal.pone.0170550.

19. Santos DN, Sousa SNS, Silva DRS, Figueiredo MLF. Percepção do idoso sobre a vacina contra a influenza. Enferm Foco [Internet]. 2011;2(2):112-5. Disponível em: http://revista.cofen.gov.br/index.php/ enfermagem/article/viewFile/107/89

20. Neves RG, Duro SMS, Tomasi E. Influenza vaccination among elderly in Pelotas-RS, Brazil, 2014: a population-based study. Epidemiol Serv Saúde [Internet]. 2016;25(4):755-66. Disponível em: http://10.5123/S1679-49742016000400009.

21. Pinto CJM, Pereira EHR, Teodoro CM, Becari RA, de Assis CG, Ferrari JC, et al. Vaccination against influenza in elderly people: factors associated with acceptance and refusal of the vaccine. Rev Soc Bras Med Trop [Internet]. 2019;52:e20180366. Disponível em: https://doi.org/10.1590/0037-8682-0366-2018.

22. Francisco PMSB, Borim FSA, Neri AL. Vacinação contra influenza em idosos: dados do FIBRA, Campinas, São Paulo, Brasil. Ciênc Saúde Colet [Internet]. 2015;20(12):3775-86. Disponível em: https://doi.org/10.1590/1413812320152012.19702014.

23. Araújo TME, Lino FS, Nascimento DJC, Costa FSR. Vacina contra influenza: conhecimentos, atitudes e práticas de idosos em Teresina. Rev Bras Enferm [Internet]. 2007;60(4):439-43. Disponível em: http:// dx.doi.org/10.1590/S0034-71672007000400015.

24. Lobiondo-Wood G, Haber J. Pesquisa em enfermagem: métodos, avaliação crítica e utilização. $4^{\mathrm{a}}$ ed. Rio de Janeiro: Guanabara Koogan; 2001.

25. Greenland S. Model-based estimation of relative risks and other epidemiologic measures in studies of common outcomes and in case-control studies. Am J Epidemiol [Internet]. 2004;160(4):301-5. Disponível em: https://doi.org/10.1093/aje/kwh221.

26. Scutari M. Bayesian Network Constraint-Based Structure Learning Algorithms: Parallel and Optimized Implementations in the bnlearn R Package. J Stat Softw [Internet]. 2017;77(2):1-20. Disponível em: http://dx.doi.org/10.18637/jss.v077.i02.

27. Knuppel S, Stang A. DAG program: identifying minimal sufficient adjustment sets. Epidemiology [Internet]. 2010;21(1):159. Disponível em: http://doi. org/10.1097/EDE.0b013e3181c307ce.
28. Brasil. Portaria $\mathrm{N}^{\circ} 2.436$, de 21 de setembro de 2017. Aprova a Política Nacional de Atenção Básica, estabelecendo a revisão de diretrizes para a organização da Atenção Básica, no âmbito do Sistema Único de Saúde (SUS). Diário Oficial da União. 2017. Disponível em: https://bvsms.saude.gov.br/bvs/ saudelegis/gm/2017/prt2436_22_09_2017.html.

29. Brasil. Portaria no 930 , de 15 de maio de 2019. Institui o Programa "Saúde na Hora", que dispõe sobre o horário estendido de funcionamento das Unidades de Saúde da Família, altera a Portaria no 2.436/GM/MS, de 2017, a Portaria de Consolidação n ${ }^{\circ}$ 2/GM/MS, de 2017, a Portaria de Consolidação n ${ }^{\circ}$ 6/GM/MS, de 2017, e dá outras providências. Diário Oficial da União. 2019. Disponível em: http://bvsms.saude.gov.br/bvs/ saudelegis/gm/2019/prt0930_17_05_2019.html

30. Kwon DS, Kim K, Park SM. Factors associated with influenza vaccination coverage among the elderly in South Korea: the Fourth Korean National Health and Nutrition Examination Survey (KNHANES IV). BMJ Open [Internet]. 2016;6:e012618. Disponível em: https://doi.org/10.1136/bmjopen-2016-012618.

31. Gerst-Emerson K, Jayawardhana J. Loneliness as a Public Health Issue: the impact of loneliness on health care utilization among older adults. Am J Public Health [Internet]. 2015;105:1013-9. Disponível em: https://doi.org/10.2105/AJPH.2014.302427.

32. Dardalas I, Pourzitaki C, Manomenidis G, Malliou F, Galanis P, Papazisis G, et al. Predictors of influenza vaccination among elderly: a cross-sectional survey in Greece. Aging Clin Exp Res [Internet]. 2020;32(9):1821-8. Disponível em: https://doi. org/10.1007/s40520-019-01367-4.

33. Machado A, Santos AJ, Kislaya I, Larrauri A, Nunes B. Understanding influenza vaccination among Portuguese elderly: the social ecological framework. Health Promot Internation [Internet]. 2020;35(6):1427-40. Disponível em: https://doi. org/10.1093/heapro/daaa011.

34. Instituto Brasileiro de Geografia e Estatística. PNAD Contínua TIC 2017: Internet chega a três em cada quatro domicílios do país [Internet]. Brasília, DF: IBGE; 2017 [acesso em 17 mar. 2021]. Disponível em: https://agenciadenoticias.ibge.gov.br/agencia-sala-deimprensa/2013-agencia-de-noticias/releases/23445pnad-continua-tic-2017-internet-chega-a-tres-emcada-quatro-domicilios-do-pais

35. Silva SPC, Menandro MCS. Representações de idosos sobre a vacina da gripe Ciên Saúde Colet [Internet]. 2013;18(8):2179-88. Disponível em: http://10.1590/ S1413-81232013000800002. 